\title{
Efficiency of synthetic melatonin in comprehensive therapy of patients with a combination of irritable bowel syndrome with constipation, arterial hypertension and obesity
}

\author{
Vasyl H. MISHCHUK ${ }^{1}$, Galina V. GRYGORUK ${ }^{1}$, Natalia V. HUBINA², \\ Halyna V. KOZINCHUK ${ }^{1}$, Hanna Y. STUPNYTSKA ${ }^{3}$
}

\begin{abstract}
${ }^{1}$ Department of General Practice (Family Medicine), Physical Rehabilitation and Sports Medicine, Ivano-Frankivsk National Medical University, Ukraine

${ }^{2}$ Department of Clinical Pharmacology and Pharmacotherapy, Ivano-Frankivsk National Medical University, Ukraine

${ }^{3}$ Department of Internal Medicine and Infectious Diseases, Bukovinian State Medical University,

\begin{abstract}
Introduction. The problem of pathology of internal organs comorbidity with obesity has become particularly relevant in recent years. The association of obesity with gastrointestinal pathology, in particular, irritable bowel syndrome has not been studied enough. The role of melatonin in the development of these diseases and the possibility of using its synthetic forms in their treatment has drawn the attention of researchers in recent years.

Objective - to study the effect of synthetic melatonin on blood pressure, autonomic status, sleep quality, the level of ghrelin and serotonin in patients suffering from obesity combined with arterial hypertension and irritable bowel syndrome with constipation.

Material and methods. The study involved 78 patients, of which 34 were diagnosed with irritable bowel syndrome with constipation combined with obesity, and 54 of those who suffered obesity, irritable bowel syndrome combined with arterial hypertension. Patients of each group were divided into two subgroups depending on the treatment complex, one of which received standard therapy, and in the case of combination with arterial hypertension, they also received three component antihypertensive therapy, while the other two received synthetic melatonin before the standard therapy.

Results. There was a more significant decrease in average daily systolic and diastolic blood pressure values under the influence of therapy with the inclusion of vitae-melatonin and a significant decrease in the number of patients with a non-dipper and night-picker blood pressure profile. The more positive influence of comprehensive therapy with the inclusion of melatonin on parameters of heart rate variability and its quality was noted. The administration of melatonin combined with a standard therapy led to an increase in the frequency of the bowel movement, approaching serotonin in the serum to normal rates and a decrease in the postprandial level of ghrelin without changes in its fasting concentration.

Conclusions. The obtained results are indicative of the positive effect of melatonin on obesity combined with irritable bowel syndrome with constipation and arterial hypertension.
\end{abstract}

Keywords: obesity, irritable bowel syndrome, arterial hypertension, melatonin 


\author{
Abbreviations \\ IBSC - irritable bowel syndrome with constipation \\ $\mathrm{AH}$ - arterial hypertension \\ BP - blood pressure \\ BMI - body mass index
}

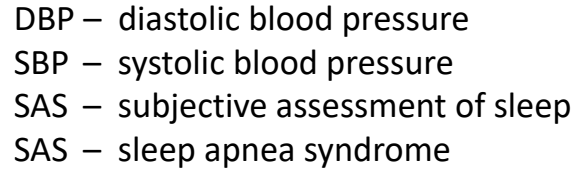

DBP - diastolic blood pressure

SAS - subjective assessment of sleep

sAS - sleep apnea syndrome

\section{INTRODUCTION}

The problem of combining the pathology of internal organs with excess body weight and obesity has become particularly relevant in recent years [1]. According to the authors obesity was diagnosed in 603.7 million adults and 107.7 million children in 2015. Also, more than $2 / 3$ deaths in overweight people were associated with cardiovascular disease. The number of overweight people in Europe has increased by three times over the past 20 years. Without its prophylaxis and treatment, only in Eastern European countries the number of obese patients will have increased to $37.0 \%$ for men and to $31.4 \%$ for women by 2030 [2]. Obesity is a background which significantly increases the incidence of coronary heart disease, arterial hypertension, type II diabetes and liver disease (fatty liver, nonalcoholic steatohepatitis) [3].

In addition, obesity is often combined with diseases of the gastrointestinal system (gastro-esophageal reflux disease, diseases of gallbladder, pancreas and intestines). According to E.A. Panova et al. [4] a combination of obesity with dyskinesia of the colon, diverticular disease, and polyposis of the large intestine were diagnosed in 36.28; $28.0 ; 10.0 \%$ of patients respectively. A more frequent combination of obesity and irritable bowel syndrome with constipation (IBSC) was also found by M. Aasbrenn et al. [5]. The prevalence of IBS in obese patients varies in the studied population and ranges from 11.6 to $24.0 \%$ in adults [6]. At the same time, F. Rusu [7] did not find any difference in the prevalence of non-infectious IBS in patients with or without obesity, although the effect of drug therapy in patients with combined pathology was lower, which also makes the problem relevant.

Both general and abdominal obesity contribute to the more frequent development of hypertension [8]. According to the authors, the incidence of hypertension in patients with abdominal obesity is very high and constitutes $47.18 \%$, whereas in patients with normal body mass, an increase in blood pressure (BP) was found in $17.75 \%$ of the individuals. In people with general obesity, hypertension was diagnosed in $70 \%$ or above of cases in Asia, above $80 \%$ in Europe and slightly less in North and South America [9]. In cases when this comorbid pathology was also combined with type II diabetes, high blood pressure was diagnosed even more frequently [10].

The causes of obesity include: eating disturbances, stress, changes in the regulatory influence of neurohormones and hormones including serotonin, ghrelin, leptin and adiponectin [11].

In recent years, the role of melatonin, which is a common chronobiotic, has been studied. It is able to regulate metabolic processes in obesity in such a way that the phase of its activity is associated with high sensitivity to insulin, and when food is not taken, it is synchronized with insulin resistance [12]. In addition, melatonin is responsible for establishing an adequate balance of energy, due to the regulation of its flow from adipose tissue. It also reduces the severity of oxidative stress and increases the level of adiponectin and reduces the amount of leptin in the blood serum [13]. Melatonin receptors were identified in the central and peripheral nervous system, in the walls of the vessels, and melatonin is involved in autonomic regulation of blood pressure, both in experiments with animals and in healthy people and in patients with hypertension, although the exact mechanisms by which it causes a hypotensive effect require further studying [14]. In animals, pinealectomy causes an increase in blood BP, while the administration of ramipril in a dose of $10 \mathrm{mg}$ leads to a decrease in both systolic and diastolic $\mathrm{BP}$, levels of malonic aldehyde and the growth of melatonin-hydroxyl melatonin sulfate metabolite [15]. In addition, melatonin is involved in the regulation of circadian oscillations of BP, has meteoric and magnetoprotective properties [16]. Also, according to N.R. Brinn and M.A. Gandhi [17], under the influence of melatonin, the transit velocity of the colon increases, the intensity of pain in the intestine decreases.

Therefore, considering the important role of melatonin in the development of obesity, hypertension and IBSc, it is important to study its internal use in such a comorbid pathology.

\section{OBJECTIVE}

To study the efficiency of the course internal intake of melatonin (vitae-melatonin, JSC Kyiv vitamin factory) on blood pressure values, vege- 
tative status, sleep quality, ghrelin and serotonin levels in patients suffering from obesity combined with arterial hypertension and irritable bowel syndrome with constipation.

\section{MATERIALS AND METHODS}

The study involved 78 patients including 24 who were diagnosed with IBSC according to Rome IV criteria combined with obesity, and 54 patients with IBSC combined with the second degree $\mathrm{AH}$, 2-3 degrees (38-II and 16-III stages) and obesity $\left(\mathrm{BMI}-36.3 \pm 0.7 \mathrm{~kg} / \mathrm{m}^{2}\right)$. Depending on the method of treatment, the patients of both groups were divided into two subgroups: in the first one 12 patients received a baseline therapy, which included dietary nutrition with mainly vegetables and fruit, a probiotic (30 $\mathrm{mg}$ of duphalac in the morning) and prokinetic (domperidone) $10 \mathrm{mg}$ three times a day before meals, atorvastatin $-20 \mathrm{mg}$ per day, while in the second one 12 patients in adition to the baseline therapy received vitae-melatonin in a dose of $3 \mathrm{mg} 30$ minutes before their usual time of sleep for three weeks. Patients in the second group were also divided into two subgroups, one of which included 16 people who received a combination therapy like the previous patients and an antihypertensive one, which included the angiotensin converting enzyme inhibitor - lisinopril in a dose of $12.0+-1.48 \mathrm{mg} /$ day, amlodipine $-10 \mathrm{mg}$, indapamide $-2.5 \mathrm{mg}$ and atorvastatin $-20 \mathrm{mg} /$ day, and the second subgroup (38 patients) - the same combination therapy with $3 \mathrm{mg}$ vitae-melatonin 30 minutes before going to sleep for 3 weeks.

All patients underwent daily monitoring of blood pressure before and after the treatment by means of the apparatus "Cardiosens AT" (manufactured by the M.U. Zhukovskyi National Aeronautical University, Ukraine). The protocol included the measurement of blood pressure every 15 minutes in the daytime from 06:00 to 23:00 and every 30 minutes at night (from 23:00 to $06: 00$ ). Studies with more than 50 qualitative measurements during the day were considered to be effective. The average daily pressure (BPa), average daytime pressure (BPdt), average night-time pressure (BPnt), hypertension time index (Hidx) for each study were calculated using the computer program installed in this device) $-\%$ of measurements that exceeded norm (at BP $>50 \%$ ), the value of the morning increase in blood pressure and the daily index (DI). The patients with the degree of reduction of BPa and BPdt overnight compared with the daytime is $10-20 \%$ were considered dipper, less than $10 \%$ non-dipper, over $20 \%$ over-dipper) and $0 \%$ - night-pearker. We also analyzed the heart rate variability (HRV) by means of the program "System: CardioLab +"XAUMEDUKA" (Kharkiv) calculating time and spectrum values, in particular LF (ms2) - power in the range of low frequencies (0.04-0.15 Hz) which characterizes mostly sympathetic activity and HF (ms2) - power in the high frequency range $(0.15-0.40 \mathrm{~Hz})$, which reflects the parasympathetic effect on sinus node activity and $\mathrm{LF} / \mathrm{HF}$ - power ratio in the low frequency range to power in the range of high frequencies, as a measure of the balance of sympathetic and parasympathetic effects on the activity of the sinus node.

To assess sleep disturbances, we collected a somnological history, analyzed a point assessment questionnaire for sleep subjective signs (SSS), an apnea syndrome during sleep screening questionnaire, and the Epworth sleepiness scale (18). The subjective characteristics of sleep, which were evaluated in a five-point system, included the time of falling asleep and the duration of sleep, the number of night awakenings and dreams, the quality of sleep and morning awakening, allocating three areas of values 22 points and above the sleep is estimated as normal, from 21 up to 19 points - boundary condition, and 18 and below the sleep is disturbed. The value of Epworth scale, which is a simple and reliable method for assessing the severity of daytime sleepiness, was assessed in points, and the index from 0 to 10 reflects normal values, 11-15 - excessive daytime sleepiness, and 16-24 - pronounced daytime drowsiness. Sleep apnea syndrome (SAS) screening questionnaire included the most frequent, specific and highly specific symptoms of this condition, the first group of which were snoring, daytime sleepiness, morning headache and increased blood pressure above 140 systolic and under $90 \mathrm{mmHg}$ of diastolic was estimated as 1 point. The second group of symptoms, including increased morning diastolic pressure over the evening one and diastolic pressure above $100 \mathrm{mmHg}$ rated as two points. The third group (stopping breathing during sleep and having a daytime drowsiness in a wakeful state - three points). If the patient receives 4 or more points, then he can be attributed to the group of patients with apnea with $96 \%$ probability. All of the above parameters of sleep quality assessment were determined before and after the course administration of vitae-melatonin. The rate of serotonin and ghrelin in the serum was measured in all patients before and after the treatment by means of sets "Serotonon ELISA IBL International GMBH" (Germany) and RAY Biotech Human Ghrelin EIA (USA) respectively.

The results were statistically analyzed using the application package Statistica 8.0 for Windows 
and the package of statistical functions of the Microsoft Excel program. The mean values and their standard errors $(M \pm m$, where $M$ is the mean value, $m$ is its standard error) were calculated for the groups. Non-parametric Mann-Whitney criteria for independent samples were used to compare the mean values. Correlations were estimated by the correlation and Spearman coefficient $(r)$. The reliability of changes in the values before and after the treatment was established using the non-parametric Wilcoxon criterion for related samples. The results where the significance level $P$ did not exceed 0.05 were considered to be reliable.

\section{RESULTS}

It has been established that inclusion of vitae-melatonin in the comprehensive antihypertensive therapy contributed to a decrease in average daily systolic blood pressure (SBP) by $42.7 \mathrm{mmHg}$ (from 187.0 \pm 2.8 to $144.3 \pm 2.1 \mathrm{mmHg}, p<0.05$ ), while under the influence of standard therapy without vitae-melatonin it decreased by $37.7 \mathrm{~mm}$ $\mathrm{Hg}$ (from $183.5 \pm 1.9$ to $145.8 \pm 1.4 \mathrm{mmHg}$ ). The average daily diastolic blood pressure (DBP) also decreased more significantly when vitae-melatonin was added to three-component therapy - by 12.3 $\mathrm{mm} \mathrm{Hg}$, (from $96.8 \pm 1.9$ to $84.5 \pm 1.0 \mathrm{~mm} \mathrm{Hg}$ ), and in patients who did not receive it - by $9.7 \mathrm{~mm} \mathrm{Hg}$ (from $97.5 \pm 1.6$ to $87.8 \pm 1.1 \mathrm{~mm} \mathrm{Hg}$ ). In patients with a combination of obesity and IBSC without significant increase in blood pressure under the influence of vitae-melatonin, the average daily ABP decreased by $19.0 \mathrm{~mm} \mathrm{Hg}$ (from 135.7 \pm 3.0 to $116.7 \pm 2.4 \mathrm{~mm} \mathrm{Hg}),(p<0.05)$, and under the in- fluence of basic therapy - by $13.9 \mathrm{~mm} \mathrm{Hg}$ (from $136.9 \pm 0.7$ to $123.0 \pm 2.7 \mathrm{~mm} \mathrm{Hg}$ ). The average daily BP decreased by 10.2 and $6.8 \mathrm{~mm} \mathrm{Hg}$ respectively (from $101.1 \pm 2.3$ to $90.9 \pm 1.9$ and from $105.2 \pm 3.1$ to $98.4 \pm 2.5 \mathrm{~mm} \mathrm{Hg}),\left(p^{1,2}<0.05\right)$.

The results of the evaluation of the daily profile of blood pressure in patients in the main group indicate a decrease in their number with the non-dipper profile from 50.0 to $10.5 \%$, while in patients in the group who did not receive vitae-melatonin - from 43.8 to $37.5 \%$. The number of patients with a daily profile of BP-night-picker after treatment with the inclusion of vitae-melatonin decreased from 12.1 to $5.3 \%$ of cases, and among those receiving baseline therapy, their number remained unchanged $(12.5 \%$ and $12.7 \%$ before and after the treatment).

The LF heart rate variability (HRV) component, reflecting the power in the low-frequency range and characterizing the sympathetic activity of the autonomic nervous system, in obese patients with IBSc exceeded the similar index in healthy people by 1.3 times, and in cases of combined with $\mathrm{AH}-$ by 1.5 times (Fig. 1). After the standard therapy in patients with obesity and IBSc, this rate of HRV decreased by $13.3 \mathrm{~ms}^{2}$, and when vitae-melatonin was administered - by $25.9 \mathrm{~ms}^{2}$. In patients with obesity, IBSC and BP, the LF index decreased by $20.9 \mathrm{~ms} 2$ (from $178.2 \pm 4.2$ to $156.3 \pm 5.4 \mathrm{~ms}^{2}$ ) due to baseline therapy, while with the addition of vitae-melatonin - by $27.2 \mathrm{~ms}^{2}$. The HF HRV component, reflecting parasympathetic effects and reduced before the treatment by 9.6 and $29 . \mathrm{ms}^{2}$, increased after the treatment in patients from the first and second groups (Figure 1). In particu-

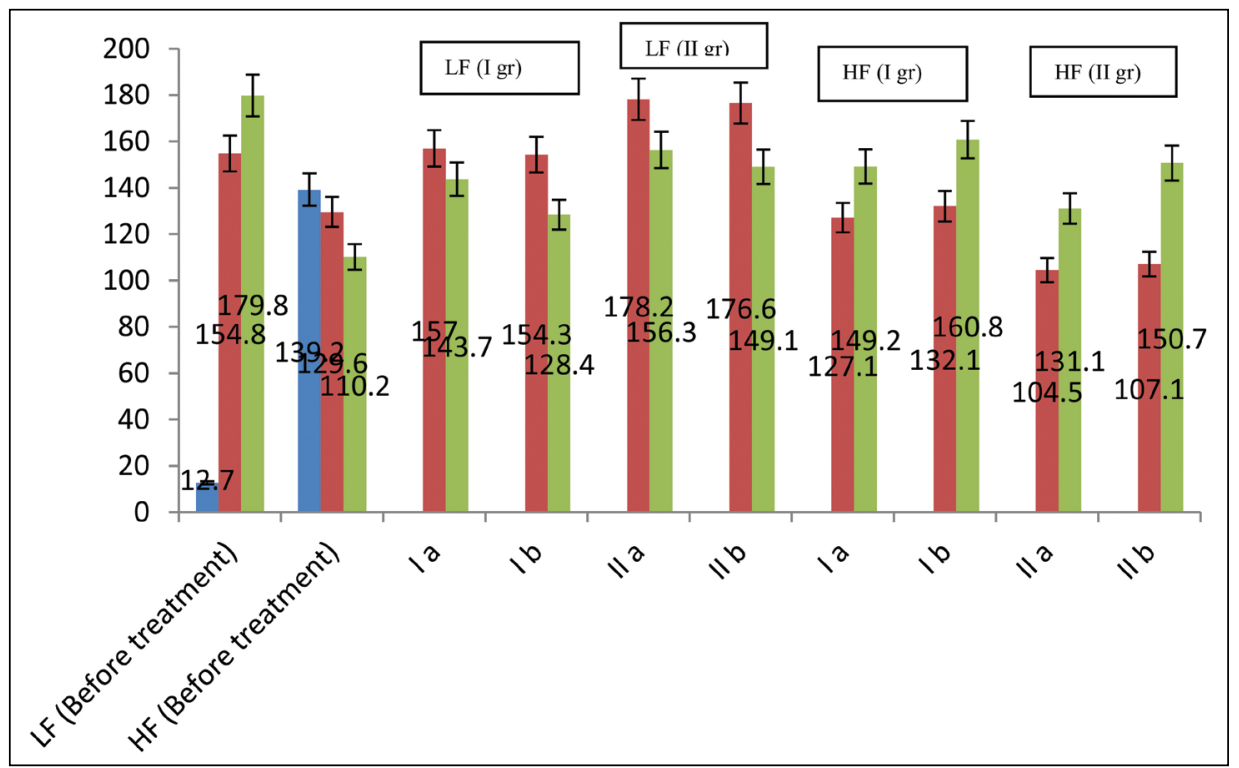

FIGURE 1. The dynamics of heart rate variability after the standard therapy and when vitae-melatonin was administered in different groups of patients 
lar, in patients with obesity and IBSc it increased, both under the influence of baseline and comprehensive therapy with melatonin. In patients with concomitant blood pressure, the component of HF HRV increased by $26.6 \mathrm{~ms}^{2}$ due to standard comprehensive therapy, and with added vitae-melatonin - by $43.6 \mathrm{~ms}^{2}$. The ratio of LF/HF in patients with a combination of three pathologies decreased from $1.42 \pm 0.05$ to $1.34 \pm 0.07$ under the influence of baseline therapy and from 1.74 \pm 0.05 to $1.04 \pm 0.06(p<0.05)$ after the therapy with the inclusion of vitae-melatonin.

Analysing the values of subjective assessment of sleep quality in obese patients with IBSc indicates that under the influence of standard therapy, it did not change $(17.8 \pm 1.1$ before and $16.5 \pm 1.0$ points - after), and with vitae-melatonin, the subjective assessment of sleep quality increased from $17.2 \pm 0.8$ to $21.1 \pm 1.0$ points. In patients with comorbidity in the form of obesity, IBSc and BP who received three component antihypertensive therapy, the sleep quality index increased by 1.2 times (from $13.7 \pm 0.8$ to $16.3 \pm 0.7$ points $(p<0.05)$, and with inclusion of vitae-melatonin - by 1.45 times (from $12.8 \pm 0.5$ to $18.6 \pm 0.5$ points, $p<0.01$ ). The results of the Epworth scale of sleep show the absence of their dynamics in patients with obesity combined with IBSc under the influence of standard therapy $(11.8 \pm 0.8-$ before and $10.0 \pm 0.7$ points - after the treatment) and some decrease in the total value (from $10.8 \pm 2.0$ to $8.8 \pm 1.2$ points) with the addition of vitae-melatonin. In patients with a combination of obesity and IBSC and $\mathrm{AH}$, who received vitae-melatonin with standard therapy, the Epworth scale of sleep indices were close to the optimal ( $14.5 \pm 0.7$ - before and $11.2 \pm 0.5$ points after the treatment), while after a standard therapy without vitae-melatonin, they decreased as well (from $15.9 \pm 1.0$ to $13.9 \pm 0.8$ points). The results of the SAS screening also confirmed the positive effect of vitae-melatonin on reducing the apnea frequency during sleep, as evidenced by a decrease in its score from $4.0 \pm 0.3$ to $2.0 \pm 0.2$ points, while in case of only basic antihypertensive therapy administration, this index has not changed $(4.6 \pm 0.4$ - before and $4.6 \pm 0.3$ - after the treatment). The positive effect of melatonin on the quality of sleep is also confirmed by the results of changes in the indices of SAS screening in patients with obesity and IBSC without concomitant $\mathrm{AH}$, under the influence of which they changed in total from 2.6 \pm 0.5 to $1.4 \pm 0.2$ points, in the absence of changes in patients of the control group receiving baseline therapy (2.4 \pm 0.5 - before and $2.1 \pm 0.5$ - after $)$.
Under the influence of integrated therapy with the inclusion of vitae-melatonin in patients with obesity, IBSC and $\mathrm{AH}$, increased before the treatment serotonin level in blood decreased from $165.5 \pm 7.5$ to $125.6 \pm 7.6 \mathrm{ng} / \mathrm{mg}$ ( $p<0.05)$, while under the influence of standard therapy, its concentration has not significantly changed (170.4 \pm 5.0 before and $163.4 \pm 4.7 \mathrm{ng} / \mathrm{ml}$ - after (in healthy persons serotonin level in blood was equal to $39.1 \pm 3.0 \mathrm{ng} / \mathrm{ml}$ ). In these patients, an increase in the frequency of evacuations from $2.2 \pm 0.2$ to $4.1 \pm 0.2$ episodes per week was also established in the absence of a change in its multiplicity under the influence of therapy without melatonin (2.4 \pm 0.2 and $1.9 \pm 0.2$ times a week). Prescribing vitae-melatonin as a component of complex therapy for patients with obesity, IBSc, and $\mathrm{AH}$ also contributed to a decrease in the postprandial level of ghrelin from $24.87 \pm 1.79$ to $18.53 \pm 1.41 \mathrm{pg} / \mathrm{ml}$ $(p<0.05)$, while under the influence of standard therapy, this index only tended to decrease. The fasting serum ghrelin level in patients with obesity and IBSc has not changed $(16.7 \pm 1.71$ - before and $16.9 \pm 1.44 \mathrm{pg} / \mathrm{ml}$ - after, and in patients receiving melatonin, increased from $17.3 \pm 1.86$ to $26.7 \pm 2.01$ $\mathrm{pg} / \mathrm{ml}$.

\section{DISCUSSION}

The established significant antihypertensive effect of the treatment with the inclusion of vitae-melatonin is to some extent consistent with the research findings of E. Grossman et al. [19], which showed that one of the possible ways of the positive melatonin effect is a decrease in a nighttime hypertension, in the absence of a significant influence of melatonin on the descrease of daytime blood pressure. Our established decrease in the number of non-dipper and night-picker patients in the group receiving vitae-melatonin may be one of the directions of its positive action. The violation of vegetative regulation at the expense of a more significant decrease in the heart rate variability in the high frequency range may also be one of the mechanisms of the IBSc development [20].

The more pronounced decrease in the LF HRV component and the increase by $43.6 \mathrm{~ms} 2$ of the $\mathrm{HF}$ component and the improvement of the LF/HF ratio are likely to be explained by the positive influence of vitae-melatonin on the state of vegetative regulation. The expediency of including melatonin preparations into the complex of antihypertensive therapy is also confirmed by C.M. Drago et al [21], according to the results of their experimental 
studies, a more positive effect of irbesartan and melatonin on individual lipidogram characterictics, blood glucose levels, and antioxidant potential enhancement of the body under arterial hypertension have been established. Melatonin may intensify the antihypertensive effect of other medications by activating antioxidant enzymes and increasing the activity of mitochondrial vascular endothelial systems and by interacting with the central nervous system [22].

Violations of quality and duration of sleep, which are most common in recent years, decreasing with an average of 1-2 hours in case of obesity, an increase in the activity of the sympathetic nervous system, which is confirmed by this study, and changes in the secretion of melatonin, are considered by a number of researchers as predictors of development of $\mathrm{AH}$ [23]. In addition, other diseases to which IBS belongs, are associated with sleep disturbances, the probability of their combination is 2.618 (95 DJ 2.052\%, $\mathrm{K}-3.341 \%$ ), and among the factors contributing to such a combination changes in vegetative regulation and intestinal microbiocenosis [24]. Sleep disturbance in patients with IBSc also correlates with the severity of pain, lower quality of life, and such effects extend beyond the intestine [25].

The very changes in vegetative regulation and its improvement after involving the standard integrated therapy of vitae-melatonin, confirmed in these research, are likely to contribute to improving the quality of sleep due to the results of its assessment. The approximation to the optimal indices of the Epworth sleep scale also confirms the effectiveness of the course of application of vitae-melatonin. The decrease in sleep apnea, according to the results of its score, obtained in this study, may also lead to a decrease in the number of patients with daily non-dipper and night-picker profiles by $39.5 \%$ and $6.8 \%$. The obtained effects are also important because the severity and frequency of obstructive sleep apnea correlates with the degree of hypertension [26]. The importance of improving the quality of sleep and body weight correction to reduce the number of patients with a daily non-dipper BP profile is also revealed in other studies [27].

Preserving the level of serotonin within the limits of the physiological norm provides a rational type of food behavior and circadian rhythms of the ghrelin secretion [11]. Reducing the level of postprandial ghrelin in blood serum and the absence of changes in its basal concentration should be considered as a positive effect, since on the one hand, ghrelin reduces the threshold of pain in the intestine [28], and, on the other hand, improves the endothelial function by increasing the bioavailability of nitric oxide [29], reduces appetite and inhibits weight gain.

\section{CONCLUSIONS}

To sum it up, the results obtained in this research regarding the positive effects of vitae-melatonin and individual components of the formation of comorbid pathology in the case of a combination of obesity, irritable bowel syndrome with constipation and arterial hypertension are stipulated by the potentiation of antihypertensive, vegetative modulatory, anti-obstipation effect, since the basis of the development of such a comorbid pathology lie the common pathogenetic mechanisms.

Conflict of interest: none declared Financial support: none declared

\section{REFERENCES}

1. Gregg EW, Shaw JE. Health Effects of Overweight and Obesity in 195 Countries over 25 Years. The G.B.D 2015 Obesity Collaborators / N. Eng J. Med. 2017; 373:13-37.

2. Krzystoszek G, Wierzegska E, Lielinska A. Obesity: An analysis of epidemialodical and prognostic research. Arch. Med. Sci. 2015; 11(1):24-33.

3. Wong E, Tanamas SK, Wolfe $R$ et al. The Role of obesity duration on the assotiation between obesity and risk physical obesity. Obesity. 2015; 23(2):443-447.

4. Panova El, Martyishina OV, Danilov VA. Pathology associated with obesity: freguency, nature and some mechanism of formation. (Published in Russian).
Sovremennye tehnologii v meditsine. 2013; 3(2):108-110.

5. Aasbrenn M, Hedestol J, Eribe $\mathrm{J}$ et al. Prevalence and predictors of irritable bowel syndrome in patients with morbid obesity a cross-sectional. BNS Obes. 2017; 4:1-8.

6. Pickket-Blakely O. Obesity and Irritable Bowel Syndrome: A. Comprehensive Rewiew. Gastroenferology and Hepatology. 2014; 10(7):411-6.

7. Rusu Flavin. Obesity and irritable bowel syndrome: coincidence or association. Gastroinfestin.Liver Dis. 2018; 27(2):20012005.

8. Sur G, Sur M, Kudor-Szabadi L, Sur L, Sporis D, Sur D. Arterial hypertension - prevalence of risk factors and morbid associations that increase cardiovascular risk. Journal of Clinical Medicine. Medica (Buchar) 2010; 5(1):34-40.

9. Colosia Ann D, Palencia R, Khan Sh. Prevalence of hypertension and obesity in patients with type 2 diabetus mellitus in observational studies: a systematic literature review. Diabetes Metab. Syndr. Obes. 2013; 17(6):327-38

10. Rosu M-M, Mota M. The prevalence of Arterial Hypertension in Romanian Adult Population: Results from the Predatorr Study. Romanian Journal of Diabetes Nutrition and Metabolic Diseases. 2018; 25(3):303-12

11. Alekseeva NS, Sayamina-Hvostova OI, Beloberodova EV, Koykova IA, 
Aspembitova AT. Indicators of hormonal levels in eating disorders in patients with metabolic syndrome. (Published in Russian). Lechaschiy vrach. 2017; 10:92-95

12. Cipolla-Neto GG, Amaral FG, Afeche SC, Tan DX, Reiter RG. Melatonin, energy metabolism and obesity: a review. $G$. Pineal. Res, 2014; 56 (4):371-81.

13. Szewczyk-Golec K, Rajewski P, Gackowski $M$ et al. Melatonin Supplementation Lowers Oxidative Stress and Regulates Adipokines in Obese Patients on a Calorie - Restricted Diet. Oxid Med. Cell Longey. 2017; 2017:8494107

14. Baker J, Kimpinski K. Role of melatonin in blood pressure regulation: An adjunet antihypertensive agent. Clinical and Experimental Pharmacology and Physiology. 2018; 45(8)

15. Grigore C, Stoian J, Grigore O, Brucknem JV Melatonin Level Modifacation after Ramipril Treatment for Hypertension. Conference At Prague Czech Republic, January 2011 ISBN: 978-1-61804-036-7

16. Mazdzan M, Chalubinski M, Wojdan K, and Broncel $M$. The effect of melatonin on circadian blood pressure in patients with tipe 2 diabetus and essential hypertension. Arch. Med Sei. 2014; 10(4):669-675.

17. Brinn NR, Candhi MA. A Review of the Role of Melatonin in Irritable Bowel Syndrome. American Journal of Pharmacy and Health Recearch 2014; 2(12):1-16.
18. Levin YAl, Kovrov GV, Poluektov MG et al. Insomnia: modern diagnostic and therapeutic approaches. E'jdos Media. Protocol for patients with sleep disorders. http.sleepmed.ru (Published in Russian). 2005.116

19. Grossman E, Laudon M, Zisapel N. Effect of melatonin on noctural blood pressure: meta-analisis of randomized controlled trials. Vascular Health and Risk Management. 2011; 9:7577-584

20. Mazur M, Furgala A, Jablonski K, Mach T, Thor P. Autonomic nervous system activity in conspiration-predominant irritable bowel syndrome patients. Med. Sci Monit. 2012; 18(8):493-499

21. Dragoi CM, Nicolae AG, Popa DE, Dumitrescu JB, Arsene AU, DrigoreC, Dinu-Pirvu C. Oxidative Stress and metabolic profiles assessment after melatonin and irbesafran co-administration in a novel pharmaceutical formulation. Endocrine Abstracts. 2017; 49 EP376|

22. Pechanova O, Ludovit P, Simko F. Peripheral and Central Effects of Melatonin on Blood Pressure Regulation. Int. J. Mol. Sci 2014; 15(10):17920-17937.

23. Vizir VA, Sadomov AS, Demidenko OV Duration of sleep as a risk factor for cardiovascular disease. (Published in Ukranian). Arterialna hipertenziia. 2013; 1(27).

24. Ben Wang, Rugiao Duan, Liping Duan. Prevalence of sleep disorder in irritable bowel syndrome: Asystematic review with meta-analysis. Saudi J. Gustroenterol. 2018; 24(3):141-150

25. Patel Ami, Hasak S, Casseli B, Corba MS, Vivio EE, Kumar M, Gawali C, Sayuk GS. Effects of Disturbed Sleep on Gastrointestinal and Somatic Pain Symptoms in JBS. Aliment. Pharmacol. Ther. 2016; 44(3):246-258

26. Sun-Woo Jun-Sang, Hwangbo Young, Kim Won-Joo, Chu Min Kyung, Yun Chang-Ho, Yang Kwang JK. Prevalence, Sleep Characteristics and comorbidities in a population at high risk for obstructive sleep apnea: A nationwide questionnaire study in South Korea. Plos One. 2018; 13(2):1/1414/14.

27. Tilea J, Petra D, Ardeleanu E, Hutanu A, Varga A. Clinical Conditions and Predictive Markers of Non-Dipper Profile in Hypertension. Acta Medica Marisiensis. 2018; 64(1):10-16.

28. Mao Y, Liz Chen K, Yu H Zhangs S., Jiang $\mathrm{M}, \mathrm{Ma} Y$, Liang $\mathrm{C}$ et al. Antinoceptive Effect of Ghrelin in a Rat Model of Irritable bowel Syndrome Involves TRPV1 Opioid Systems. Cell Physical Biochem. 2017; 43:518-530.

29. Tesauro M, Schinzari E, Caramanti M, Lauro R, Cardilo C. Cardiovascular and metabolic effects of ghrelin. Curr. Diabets. Rew. 2010; 6(4):228-235. 